\title{
Computer Evaluation of Restaurant Sites
}

FRANCIS R. CELLA

Professor of Business Statistics

University of Oklahoma

\section{COMPUTER EVALUATION - HOW DOES IT WORK?}

- Build the Basic Model

1. Determine the factors related to restaurant business volume.

A broad list of suggested factors can be obtained from those experienced in restaurant operation and from consultants. This list will include factors already expressed in figures area population, income, traffic volume, number of competitors, advertising budget, and so on. It will also include opinion factors which must be rated from 1 to 10 such as site visibility, neighborhood, ability of the manager, and so on.

2. Test these factors and the ratings given each with the experience of similar type of restaurants before accepting them into the model.

3. Build a preliminary mathematical model and establish the relationship of total business volume with each factor accepted. That is, determine mathematically how much each factor contributes to business success.

4. If all factors have been correctly identified and rated, the sum total of the final model will be $100 \%$. Should the sum total be less than $100 \%$, seek new contributing factors and reevaluate those previously used.

Professor Cella has built seven basic models for different types of restaurant operations-general restaurants, drive-ins, hamburger places, cafeterias, specialty restaurants, highway restaurants and certain franchise restaurants.

- Use the Model to Evaluate Similar Restaurants

To evaluate the site of a proposed new restaurant of the same type - or the potential of one already in operation - test the identifiable factors of the project being evaluated against the particular model established.

\section{- Time and Cost}

Building the basic model takes months of research time and costs thousands of dollars. Using the model to test site potential ordinarily requires only 5 minutes of computer time and a few hundred dollars.

- Computer evaluation is a new management tool, one that can be more precise than an "educated hunch." - Editor
SITE EVALUATION is probably the most important yet difficult aspect of decision-making faced by the restaurant manager who wants to invest in a new establishment or expand one already in operation. If he decides to go ahead, construction and financing costs involve large investments. If he miscalculates, he ends up with too large or too small an operation to obtain the maximum yield, and the maximum yield is typically essential to success.
To begin with, the factors that determine a site's favorableness are not too easy to identify. Once identified, they are hard to assess. Under these circumstances, it is a credit to successful restaurant operators that they have done so well in handling their site evaluation decisions. But, unfortunately, there are also examples on record of horrible miscalculations. Obviously, in the face of soaring costs for new construction, some procedure more precise than intuitive or "judgment" processes is needed. 
The computer process discussed in this article has enjoyed a record of success. It represents an application of the relatively new approach to decision-making called management science. A few years ago the mathematical procedures involved would have required untold hours of statistical manipulations, a practice far too lengthy and expensive for most restaurant operators. But with the use of today's rapid computers, answers can be obtained in minutes provided that proper information has been correctly programmed into the computer.

\section{The Computer Process}

The computer process uses a mathematical model [concept of the proposed establishment] to predict potential business volume. A mathematical model can be built for almost any type of retail establishment - restaurant, motor inn, service station, food store, etc. The model joins together all factors which influence the total volume of business, weighted according to their relative importance.

In this way, the extent to which each factor contributes to the total volume of business is measured. With this capability, it is only necessary to obtain information on each of the factors as they exist for a particular site and substitute this information in the model. The model is then processed to generate an estimate of the total volume of business for the site.

\section{Objectives of Site Evaluation}

If the objective of site evaluation is to predict the potential business volume for a certain type of restaurant on a particular site - and a decision as to the possible location will be made on the basis of this volume - then an objective process for site evaluation is available today.

\section{Factors Influencing Business Volume}

The first step, and one of the most difficult aspects of restaurant site selection, is to identify the factors which determine business volume. There are differences of opinion as to the nature and scope of these factors. However, the evidence is rather clear in conventional site evaluation processes that not all factors are considered. And those used often are not treated on the basis of their relative importance. This is partly due to inability by conventional methods of ascertaining exactly when all factors have been identified.

\section{DRIVE-IN SITE SELECTION}

For drive-in restaurants, traffic volume appears to be the most important aspect of site selection. This was the finding of NRA's 1966 Survey of Eating and Drinking Establishments, which included drive-in restaurants. When owners were asked to name their reasons for locating at their present site (96\% were in cities or suburban areas) the majority stated that traffic volume was their most important consideration. Other reasons given most often in order of preference were proximity to shopping areas, dense population, and proximity to a residential area.

But the highest average sales volume was achieved by those operators choosing visibility as their main reasons for site selection. The following table describes financial results for site location reasons:

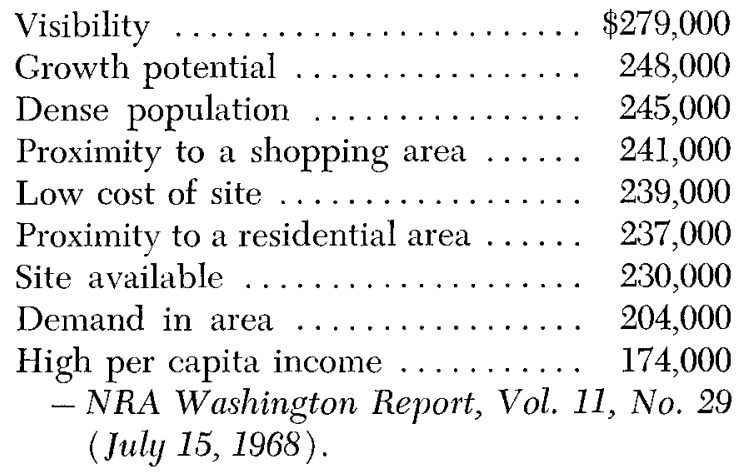

In the computer process being described, these factors are identified.

This is done first by obtaining suggestions from those knowledgeable on the subject and then by preparing a list of these suggested or hypothetical factors. A wide range of possibilities can be obtained after discussions with enough people. Eventually, the extent to which all factors have been identified and included in the analysis is determined through objective methods.

The factors group into two categories 1) quantitative and 2) qualitative.

The quantitative factors include population, income, advertising budget, number of competitors, traffic volume, and similar items which are expressed in numerical form.

The qualitative factors - such as characteristics of the neighborhood, ability of the manager, site visibility, and so on - must be quantified for use in a mathematical model. This is done by means of rating scales ranging from 1 to 10 wherein carefully defined and described choices are made available for each qualitative factor. 
Francis R. Cella served the University of Oklahoma as Director of Business Research from 1948 to 1968 and is currently professor of business statistics. Before joining the University of Oklahoma faculty, he was a statistician for the State of Kentucky and for the U.S. Army Air Corps, in which he holds a reserve commission as lieutenant colonel. Professor Cella is the author of numerous articles on business and economic research and is listed in Who's Who in America.

For more than five years Professor Cella has cooperated with the Oklahoma Restaurant Association in the development and testing of restaurant business volume by means of computer management science. He has developed models for at least seven types of restaurants and successfully used them for numerous organizations across U.S. and Canada. In April 1968, he was a featured speaker before Boston College's Fourth Annual Management Institute for Franchising. Persons interested in obtaining further information concerning Professor Cella's program should contact Mr. Justin Hill, executive director of the Oklahoma Restaurant Association, 2207 North Broadway, Oklahoma City, Okla. 73103.

The information supplied on an individual factor is eventually tested with the experience of other restaurants before being accepted for use in the model building process. This is done by means of scatter-diagrams, which quickly reveal when an item of information is in conflict with the experience of other restaurants.

\section{Sampling Existing Restaurants}

After the list of hypothetical factors has been established, information on each of them is obtained by means of a sample of operating restaurants. The sample must be a representative crosssection, or the analysis will be biased and inaccurate. Statistical techniques are available for designing samples and assuring their reliability for analysis purposes. The restaurants of the sample are surveyed to obtain their experiences with respect to each of the factors being studied.

Another difficult but significant aspect of the process is to obtain complete and accurate information on each factor from the restaurants in the sample. When the data collection is completed, the sample is used to build a model which describes how the factors affect the operations of a restaurant.

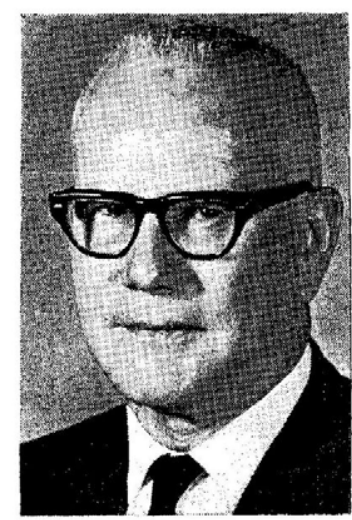

\section{Building a Preliminary Model}

The model-building process begins by establishing functional relationships between business volume and each of the factors which have been hypothesized as influencing the volume. This is done by using the information of the sample to first calculate the gross regression * between business volume and each factor.

The next move is to convert the gross regression coefficients to net regression coefficients. This is necessary because, in the gross relationship, other factors will be influencing total business volume than the one under study, which will result in an over-emphasis on the apparent impact of a factor. The net regression coefficients, by definition, measure the impact of a factor upon total business volume, with the influence of other factors eliminated by being held constant.

Why a Computer Is Needed. Converting the gross regression coefficient to net regression coefficients is a formidable task, one that would be prohibitive without the aid of a large computer. To make the conversion, the relative importance of each factor is ascertained initially. This is done by means of partial correlation coefficients, which, by definition, measure the relative importance, thus making them importance weights. To calculate the partial correlation coefficients, the gross correlations between each of the factors and between the factors and total business volume are calculated first. These gross correlations are then substituted in the appropriate formulas to calculate partial correlation coefficients.

* This is a measure of the extent to which one variable, such as sales, changes in magnitude when another variable, such as population, also changes in magnitude. The associated variation may be due to cause and effect or to coincidence, but this must be determined from the economics of the problem. 
For a model comprising fifteen factors, this task would require the time of forty clerks for about six months. But a computer program was developed which permitted the calculations to be completed in less than five minutes on a large IBM 360 computer. The gross regression coefficients are then multiplied by their respective partial correlation coefficients to obtain the desired net regression coefficients.

How to Measure a Factor's Importance. Multiplying the gross regression coefficients by their respective importance weights may change the functional relationships appreciably. If a factor exerts a significant amount of influence upon total business volume, it will have a comparatively large gross regression coefficient initially so that the impact of the importance weight will reduce this regression very little. A factor which exerts comparatively small impact, however, will have a small importance weight and the magnitude of its gross regression coefficient will be reduced appreciably. When this aspect of the process is completed, tentative estimates of the impacts of each of the factors upon total business volume will have been established.

The tentative estimates are next organized into an equation, using a symbol for each factor and the values of the net regression coefficients as the constants. The appropriate signs are introduced before each regression coefficient and the tentative model is completed. It will appear in the following format:

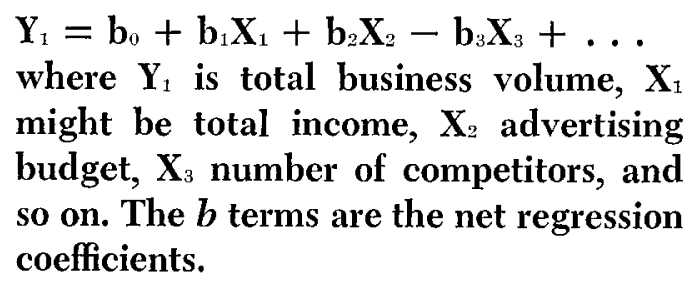

\section{Evaluating the Model}

Whether the model is performing efficiently must next be established. It is particularly important to ascertain 1) the extent to which all the factors have been identified, and 2) whether the structure of the relationships is being described correctly. The extent to which these and other conditions are reducing the efficiency of the model, can be ascertained by means of the coefficient of determination, $\mathrm{R}^{2}$. By definition, the coefficient of determination measures the degree to which the variations in business volume have been explained by the variations in the factors included in the model.

When all the factors have been identified and measured correctly, the coefficient of determination, which is a relative, will equal 100\%. When the coefficient of determination is less than $100 \%$, as is usually the case in the preliminary model, this is evidence that the model needs improvement.

\section{Developing the Final Model}

A number of actions will normally be required to develop the final site evaluation model. The first one is to determine when non-linear relationships have been treated as linear initially. For example, the impact of advertising begins to decline as a market is saturated, and this converts a linear relationship into a declining rate of increase. As the number of competitors increase, they begin to cost a restaurant less business, sometimes reaching the point where additional competitors actually contribute to an increase in business. " This means a decreasing rate of business will begin to slow-down and eventually level off. There will be other instances wherein non-linear relationships must be employed to describe a situation correctly.

After the structure of the relationships has been corrected, the basic data may require adjustment. (It is possible that this step should precede the calculation of the gross regression relationships.) These adjustments will take a variety of forms. It may be that the impacts of some factors upon business volume are delayed, which must be allowed for by appropriate lag and lead adjustments between these data and business volume. Sometimes factors affect each other as well as business volume, which may over-estimate their impacts upon total business volume. When this occurs, the impact (multicollinearity) must be removed. After these and other adjustments have been made, the data will have been purified and more truly reflect the manner in which the individual values of the factors influence business volume for a particular type of restaurant.

Another very important action may be to revise the make-up of the factors. The preliminary model comprises the factors that were hypothesized initially to be controlling total business volume. The test of the hypothesis can be heart-

"A "restaurant center" may attract a broader range of patronage for all restaurants in the complex and thus increase business. $-E d$. 
breaking, particularly when the coefficient of determination is very low. In analyzing the preliminary model, it will be found that some of the hypothetical factors have little or no influence upon business volume, and can be eliminated from further consideration. Then the search must begin for overlooked factors. This is done by a repetition of the process for building the initial model, with suggestions being obtained and the restaurants of the survey contacted again to obtain information with respect to the new factors. Then the information is processed in the same manner as that of the initial sampling.

Sometimes it will be learned that the sample was not representative and the entire model building process will have to be re-done. Not infrequently, it will be found that some of the basic data are inaccurate and new information must be obtained. Each model being built generates its own unique situations which must be handled with care and imagination. Yet, with a sufficient knowledge of economic theory, the management scientist can cope with these situations successfully.

Eventually, after enough experimentation, a model will be developed for which the coefficient of determination approximates $100 \%$. We have always been satisfied when $\mathrm{R}^{2}$ exceeds $95 \%$ because the remaining factors were of too little consequence to justify the additional effort to include them in the model. Furthermore, we were not sure that the quality of the judgment processes going into the utilization of the findings of the models will be of a $100 \%$ precision themselves.

In order to have an understanding in this connection, it was agreed at the outset of each modelbuilding contract, that the estimates of business volume would be correct within a range of plus or minus five percent, with a $95 \%$ probability of being correct. This agreement reduced the necessity of a $100 \%$ coefficient of determination, thus saving much time and money.

\section{The Final Model}

When all the processing is completed, the final model will have been obtained. This model will be an equation consisting of a large number of factors. The number of factors differs among models, with our experience varying from fifteen to twenty-four. A model can be processed for site evaluations on a computer, or with the assistance of a desk calculator. Each model was pro- grammed in Fortran IV, after employing PL/1 in one instance. In this way, a large number of site evaluations, necessary comparisons, and studies of alternatives can be expedited.

\section{Types of Models}

It was discovered early in the author's work with model building that the factors and their importance varies by type of restaurant. Therefore, five models were originally built: 1) general restaurants, 2) drive-ins, 3) hamburger places, 4) cafeterias, and 5) specialty restaurants. Later a model was built for highway restaurants. Since that time, special models have been built for a number of franchised restaurants.

Each of the models is different, both in factors and measures of the impact of the factors upon business volume. It has been discovered that the factors and their measured impact upon business volume vary in different parts of the nation so that models are being built for restaurants on a geographic basis. This program was necessary to obtain models which accurately describe the local situations and to obtain the required degree of precision in the estimates.

\section{How a Model Is Used}

The manner in which a model is used might be in order at this time. A highway restaurant site in Georgia was being evaluated. The factors which determined the business volume for a highway restaurant, and their magnitudes for the site under study, are as follows: traffic count, 14,000; highway location, 1 ; type of location, 1 ; number of signs, 2; motel nearness, 3; service station proximity, 4; appearance, 6; distance of signs, 2 miles; hours open, 14; seats, 104; parking spaces, 20; visibility, 2; competitors, 23; years of experience, 12; check average, 95 4 ; manager's rating, 6 . These factors were substituted in the highway restaurant model and multiplied by their respective regression coefficients. The results were totaled and an estimated business volume of $\$ 201,000$ to $\$ 221,000$ was obtained. This restaurant is now doing $\$ 210,000$ in annual business.

This process is repeated each time a site evaluation is made. When contrasted with the anxieties, energy, expenditures, and time consumed in making site evaluations by conventional methods, the efficiency of the approach is incontestable. The same may be said for the relative precision of the estimates obtained by both methods. Actually, a site evaluation can be obtained in a few hours 
after the required input information is supplied, which contrasts favorably with the time required for either a sample survey or an intuitive judgment. Once the model is built, the cost of an evaluation is insignificant.

\section{Experience with Models}

The models were developed originally to make site evaluations. Over four hundred such evaluations have been made, with excellent results in most instances. Whenever the estimates were outside the agreed range of variation, it was found that either the restaurant had been improperly classified and the wrong model used, or incorrect information had been supplied for use in the analysis, usually the latter. In addition to making site evaluations, the ingenious manner in which restaurant owners capitalized upon the potentials of the models was refreshing.

In one instance an owner was not satisfied with the volume of business from a drive-in restaurant on a particular site. He had the site processed through each of the available five models. The results showed that a hamburger restaurant would be most profitable on the site. The owner made the change-over and, after a shake-down period, began making more money in one month as a hamburger place than he had made in a year as a drive-in. The use of the models for this purpose was a revelation to us.

In another instance, a young man was considering the purchase of a going restaurant. The owner assured him that it was doing a high volume of business. The young man had the site processed through the appropriate model and it showed that the site would generate a relatively small volume of business. When confronted with this information, the owner was stunned. Finally, he agreed that his estimate had been based upon a few high months of business. Thus, the model saved the young man from making an unprofitable investment, although the owner subsequently unloaded the restaurant upon another unsuspecting person.

The effectiveness of the models was revealed in another way that protected some investors. In three known instances, people had sites under consideration and each was processed through the appropriate model. The findings for each site were that the volume of business would be rather small, so the investors backed off. Later, franchised operations made their own investigations of the sites and built restaurants on the basis of the findings. Each of these restaurants subse- quently closed down due to the small volume of business.

It would be possible to continue endlessly about the effective uses of the models, but this discussion should be sufficient in that respect. Some other possible uses that are either being made or contemplated will be of greater interest.

\section{Evaluating Current Operations}

A valuable use to which the models can be put is the evaluation of the operations of a going restaurant. To do this, the factors associated with the site upon which the restaurant is being operated are run through the model and the potential business volume calculated. If the estimate is in agreement with the actual volume, the management knows it is getting all the business that the site can generate. If the estimate is above the actual volume, then it is obvious that business volume can be increased by taking the appropriate actions.

When the volume is above the estimate, then management can appreciate that it is in a unique situation in comparison with the experiences of other restaurants. It can also plan that competitors will eventually recognize something is unusual about the site - and new restaurants will be built in the vicinity.

\section{Increasing Business Volume}

The potentials of the model building approach are being employed for a new type of business analysis. The objective of this program is to ascertain how restaurants can increase their business volume.

It is recognized that restaurant managers have a wide variety of alternatives for increasing their business volume. However, the extent to which each volume will actually be increased by the alternatives is not so easily ascertained. In fact, it is not possible to do so by conventional methods because unknown or unrecognized factors will be making contributions to an increase simultaneously with known factors. This will result in an over-estimate of the extent to which a factor caused business volume to increase.

The models will overcome this limitation on the analysis process because, by their nature, the net regression coefficients measure the amount by which each factor alone contributes to the total business volume. The extent of this contribution is usually found to be less than is presumed, but it is also more realistic. 
For example, if a $10 \%$ increase in advertising expenditures is accompanied by a $10 \%$ increase in business volume, the advertising manager would be inclined to argue that an investment in advertising was profitable. However, if a new industry began operating in the vicinity of the restaurant, and a competitor closed down at the time of the increase in advertising, these factors would have also contributed to the increase in business, thus reducing the extent to which the increase in advertising contributed to the increase in total business volume. Similar explanations may hold for the impact of increases in other factors upon total business volume.

Since our models measure the extent to which a change in each factor actually influences the volume of business, alternatives can be proposed and tested. Also, combinations of actions can be tested in search of the group that will bring about the greatest increase. Then the costs of each of the alternatives can be calculated and compared with the gains in business.

By employing this approach, the restaurant owner will know the additional profits that can be expected before financing any of the proposals. If he finds that increased business will be attained, yet profits reduced, it is obvious that the alternatives should not be undertaken. If some of the proposals will generate increased profits, these can be identified and employed.

Information of this type is difficult to obtain but the structure of the models makes it possible to do so. Testing the alternatives can be a difficult and time-consuming task - but the speed of the computer and the processes of management science permit the job to be done rapidly and orderly.

\section{Accuracy of the Input Data}

References made to the significance of accurate data in the evaluations are amplified here because of the significance of accuracy to the success of the process. A computer is a "dumb" beast which will perform only as it is instructed. Consequently, when a computer is given incorrect information with respect to any of the factors, it will generate incorrect information. Experience has shown that delineating the drawing area of a restaurant is a difficult task. Yet this determines the income, population and number of competitors of a restaurant, important factors in determining total business volume.
To demonstrate the effects of incorrect input information, for one report on which we worked, we found an original estimate for business volume of $\$ 313,000$. However, because a restaurant across the highway was doing only $\$ 125,000$ in business, we were suspicious of the $\$ 313,000$ estimate. We had reliable information on this competitor and used it to check the accuracy of the information supplied us. The traffic count given us was 5,200 while that for the restaurant across the highway was only 3,700 ; the parking lot had space for only 50 automobiles rather than the 200 indicated. Moreover, the restaurant across the highway had 17 competitors instead of the 7 indicated to us. When the incorrect information was replaced with actual figures, the estimated restaurant volume of $\$ 313,000$ was reduced to range from $\$ 126,000$ to $\$ 137,000$. During the course of our investigation, we learned that the evaluation was being made for a restaurant to do $\$ 127,000$ annually, thus our finding was on target.

\section{Potentials of the Process}

The model building process described is not particularly new in substance. The basic process has existed in the statistical methods area for many years under the title of multiple regression analysis. The new process contains some techniques that eliminate the danger of incorrect signs for the regression coefficients and generate accurate measures of the impacts of each factor upon business volume. Developments in electronic computers made the model-building process feasible because the volume of calculations would have been prohibitive otherwise.

A means of getting classroom knowledge into business practice was one of the most difficult obstacles to overcome, as is true for many of the other analysis processes of management science. However, we were fortunate in this respect because members of the Oklahoma Restaurant Association recognized the possibilities of the process and supplied the data and financing which made the program possible. The techniques are equally applicable in site evaluation for service stations, grocery or drug stores, dry cleaning establishments, or any other type of activity for which there are many establishments with experience that can be synthesized into a process which describes how, on the average, the individual establishments function. 